\title{
Topological and statistical approaches describing in- homogeneously polarized light fields
}

\author{
Angelsky O.V. ${ }^{1}$, Polyanskii P.V. ${ }^{1}$, Hanson Steen G. ${ }^{2}$, Ushenko A.G. ${ }^{1}$, \\ Ushenko Yu.A. ${ }^{1}$ and Angelskaya A.O. ${ }^{1}$ \\ ${ }^{1}$ Department of Correlation Optics, Chernivtsi National University, 58012 \\ Chernivtsi, Ukraine, oleg@optical.chernovtsy.ua \\ ${ }^{2}$ Optics and Plasma Research Department, Risø National Laboratory, \\ Technical University of Denmark, DK-4000 Roskilde, Denmark.
}

Received: 22.10.2007

\begin{abstract}
Relationships between the topological and statistical approaches for describing inhomogeneously polarized optical fields are considered. It is shown that the correlation length of polarization parameters of the field is comparable with the mean distance between the points with orthogonal states of polarization.
\end{abstract}

Keywords: polarization, correlation, biological tissue, statistics, singular optics

PACS: 42.25.Ja, 42.25.Lc, 47.63.Jd, 42.25.Fx.

\section{Introduction}

Interaction of coherent laser radiation with material objects results in enriching the field with information on the object's characteristics. Diverse mechanisms of this process are determined by the object's nature. Nevertheless, the object field supports the data on the structure of the object in the coordinate or spatial-frequency (angular) distributions of photometric characteristics (intensity), amplitude, phase or polarization parameters of the field [1,2], which are commonly measured in the near (Fresnel) zone or in the image plane. Under proper conditions, viz. if the spatial-frequency filtration due to aperture limitations is negligible, the field in the image plane can be considered as a scaled copy of the boundary object field, which reproduces the polarization structure of the latter.

Natural objects and structures can be classified into deterministic and random (statistical) ones. Naturally, the sets of parameters used for characterization of such structures are also different. In this paper we will analyze correlations between the topological and statistical approaches for describing polarization structure of the object fields in the image plane. As a sample for experimental study, we use biological tissues that transform considerably the state of polarization of the probing beam.

\section{Topological (singular optical) and statistical approaches}

It is known [3] that the topological approach for description of polarization structure of freely propagating object fields assumes two models, "island"-like and "nest-doll"-like 
ones. These models provide description of the structure of spatial inhomogeneity in the polarization field distribution at the local (microscopic) level. Within the "island" model one assumes that the areas with clockwise circular and elliptical polarizations are separated from the areas with counterclockwise circular and elliptical polarizations by closed contours, at which the field is linearly polarized. Along such a contour, which is often referred to as $S$ contour, the azimuth of linear polarization changes as shown in Fig. 1a. The set of the islands with clockwise polarizations and their distribution in the "ocean" of counterclockwise polarizations (or vice versa) determines the polarization structure of the field as a whole. The "nest-doll" model assumes subsequent nesting $S$ contours, which separate clockwise and counterclockwise polarizations in turns (Fig. 1b).

Polarization structure of the boundary object field and the field in the image plane may be considerably different from freely propagating field, being strongly dependent on nature of the object, in part on specific coordinate distribution of optically isotropic and optically anisotropic phases.

The problem of interest is to establish interconnection between the phenomenological approach operating within the concept of field topology and the statistical approach recently put forward in the works [4-6], where quantitative estimation of degree of interdependence (correlation) among the polarization parameters of the field at its different points is based on the degree of mutual polarization of that field. As a quantitative parameter, one uses the parameter $V$ determined as (see [6])

$$
V^{2}\left(\mathbf{r}_{1}, \mathbf{r}_{2}, \tau\right)=4 \frac{v_{1}^{2}+v_{2}^{2}+v_{3}^{2}}{I\left(\mathbf{r}_{1}, t\right) I\left(\mathbf{r}_{2}, t+\tau\right)}
$$

where $I\left(r_{1}, t\right)$ and $I\left(r_{2}, t\right)$ are the intensities of the field at the points $r_{1}$ and $r_{2}$, and the "cross Stokes parameters" are defined by the following relations:

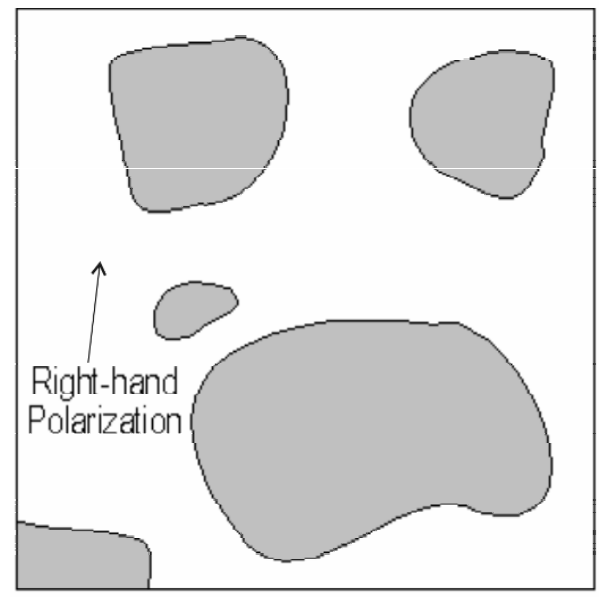

a)

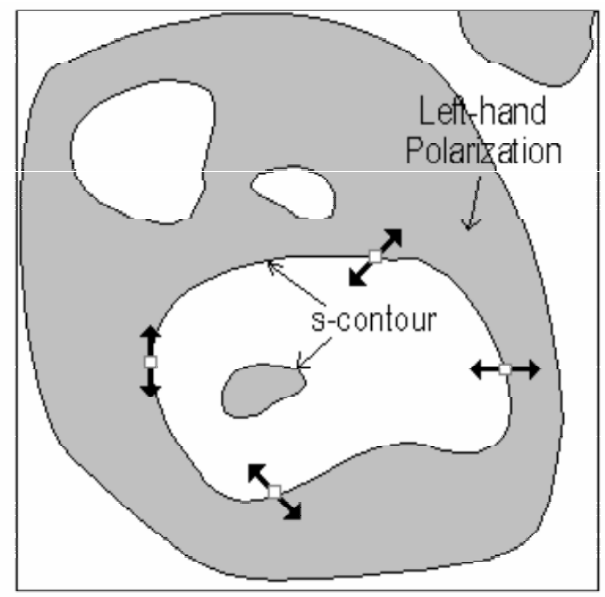

b)

Fig. 1. Typical models for describing inhomogeneity in the polarized speckle fields: "island"-like model (a) and "nest-doll"-like model (b). 


$$
\begin{aligned}
& v_{1}=\frac{<E_{x}\left(\mathbf{r}_{1} ; \tau\right) E_{x}^{*}\left(\mathbf{r}_{2} ; \tau\right)>-<E_{y}\left(\mathbf{r}_{1} ; \tau\right) E_{y}^{*}\left(\mathbf{r}_{2} ; \tau\right)>}{2}, \\
& v_{1}=\frac{<E_{x}\left(\mathbf{r}_{1} ; \tau\right) E_{x}^{*}\left(\mathbf{r}_{2} ; \tau\right)>-<E_{y}\left(\mathbf{r}_{1} ; \tau\right) E_{y}^{*}\left(\mathbf{r}_{2} ; \tau\right)>}{2}, \\
& v_{3}=i \frac{<E_{x}\left(\mathbf{r}_{1} ; \tau\right) E_{y}^{*}\left(\mathbf{r}_{2} ; \tau\right)>-<E_{y}\left(\mathbf{r}_{1} ; \tau\right) E_{x}^{*}\left(\mathbf{r}_{2} ; \tau\right)>}{2} .
\end{aligned}
$$

The indices $x$ and $y$ indicate the state of polarization, and the angular brackets denote "ensemble averaging". The algorithm for experimental determination of the magnitudes $v_{1}, v_{2}$ and $v_{3}$ suggested in Refs. [5,6] is close to the algorithm for measuring of the conventional (one-point) Stokes parameters. It consists in determining the differences between visibilities of the $x$ - and $y$-component interference patterns from the two beams for the known orientations of a quarter-wave plate and a linear analyzer. One can expect that the use of this parameter would provide a deeper understanding of interconnections between the two mentioned approaches for describing inhomogeneity in the polarization fields. Really, discussions in Refs. [4-6] have shown that the parameter $V$ (or $V^{2}$ ) is invariant in the description of inhomogeneously polarized fields.

Within the framework of model consideration, let us analyze a superposition of two waves with different states of polarization. Without any loss of generality, we first consider the case, where two superimposing waves are linearly polarized. Mutually orthogonally polarized waves, I and II, are decomposed into two orthogonal linear components in the basis $X O Y$ (see Fig. 2a). For more clarity, the $x$ and $y$ components of the two waves are shown as dotted and chain lines, respectively. One can see that the superposition of two mutually orthogonal linearly polarized waves is equivalent to the two superimposing systems of interference fringes which correspond to interference of the $x$ and $y$ components of the two waves. The two interference patterns (the dotted and chain lines) are shifted by a half spatial period, due to the fact that the $y$ components of the two waves are in phase at the points where the $x$ components have the opposite phases, and vice versa. Notice that the interference patterns for the both components in Fig. 2a have the same visibility, which differs from unity only due to different amplitudes of the two waves. One can choose a new basis $X^{\prime} O Y^{\prime}$ in such a manner that the visibilities of the two-component patterns be different. As a result, one of these patterns may be of a unit visibility. Nonetheless, due to orthogonality of the two waves, the modulation of intensity of the two patterns would be equal, so that the patterns would always be complementary, and the resulting visibility equal to zero. Thus, the orthogonal linearly polarized waves form a uniform intensity distribution with the zero visibility. In frame of the scalar statistical approach, zero visibility corresponds to a complete decorrelation of two disturbances, and in the case under interest it reveals polarization orthogonality of the disturbances with a stationary phase difference.

Ukr. J. Phys. Opt. 2007, V8, №4 


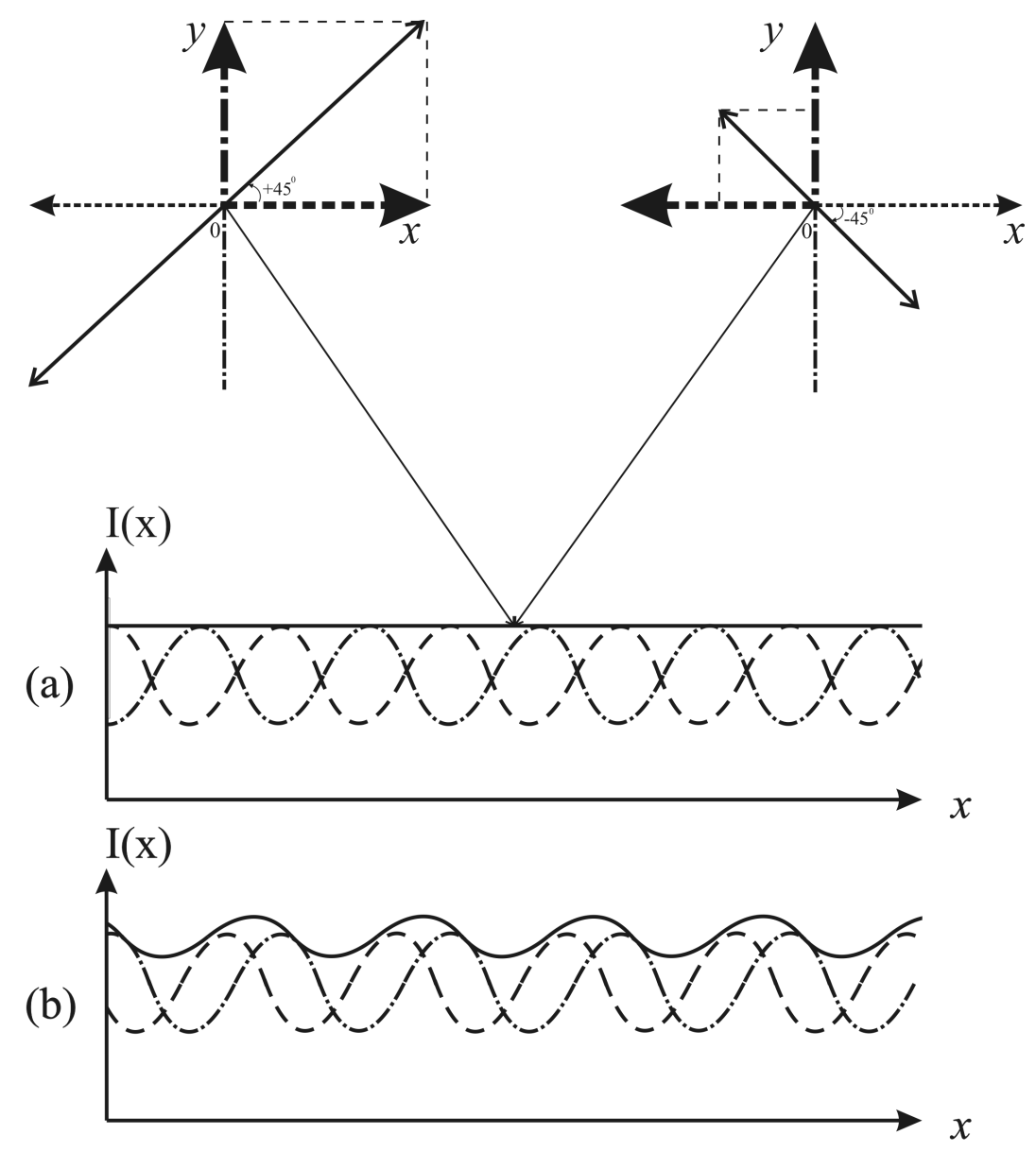

Fig. 2. Illustration of superposition of orthogonal linearly polarized beams. The above superposition is equivalent to superposition of dotted (for the $x$ component) and chain (for the $y$ component) interference patterns and results in a uniform intensity distribution at the observation plane (zero visibility - see figure, a). The phase shift in the $x$ component of beam I results in non-zero visibility of the resulting pattern, as a consequence of non-orthogonality of the beams I and II (see figure, b).

The same situation takes place if one considers a more general case of superposition of the waves with arbitrary mutually orthogonal polarization states.

Changing the phase of the $x$ or $y$ components of one of the superimposing waves (for the same amplitude ratio) brings about two waves without polarization orthogonality and results in non-zero visibility of the resulting pattern, due to a shift of one of the component patterns (see Fig. 2b).

This statement is illustrated by the following gedanken experiment. Let us consider a two-slit Young's interference experiment (Fig. 3) using quarter-wave plates and linear polarizers. Two waves are formed with equal amplitudes and orthogonal linear polarizations characterized with the azimuths $+45^{\circ}$ and $-45^{\circ}$ at the slits 1 and 2 , respectively. Assume that the paraxial approximation is valid. In the observation plane, which is parallel to the opaque screen with the slits, one observes a uniform intensity distribution. 


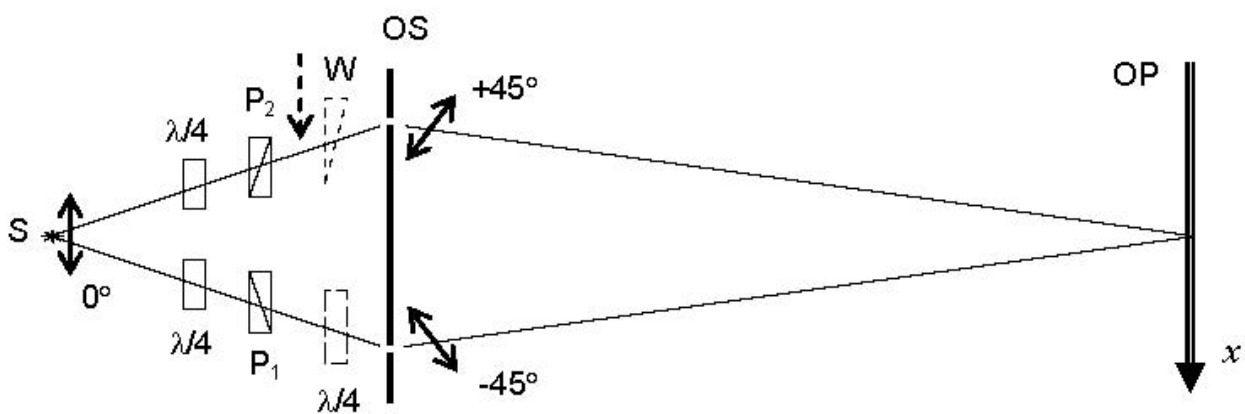

Fig. 3. Two-slit Young's arrangement of a gedanken experiment: $S$ - source of linearly ( $x$-polarized) primary source, $\lambda / 4$ - quarter-wave plates, $P_{1}$ and $P_{2}$ - linear polarizers, $O S$ - opaque screen, $O P$ - observation plane. Dashed controlled wedge $(W)$ is used for the observation of polarization modulation at the fixed point in the observation plane. Dashed quarter-wave plate is used to provide a phase shift between the $x$ and $y$ components in the lower arm of interferometer.

However, a polarization analysis would reveal periodical spatial modulation of the intensity in the plane of observation. Within the spatial period, the polarization changes gradually from horizontal linear ( $x$-polarized), through counterclockwise circular, vertical linear ( $y$-polarized) and clockwise circular, back to horizontal linear (cf. Fig. 4a). Similar polarization modulation (but now in time) is observed at the fixed point in the observation plane after introducing a controlled phase delay in one of the two waves, e.g. by using an optical wedge (see Fig. 3). For such a delay, a half-period shift between the $x$ and $y$ components is maintained, but the spatial inhomogeneity in the polarization distribution moves as a whole along the $x$ axis, which joins the two slits.

This gedanken experiment can be further complicated by introducing optically birefringent element in front of the second slit, with its fast and slow axes oriented along the $x$ and $y$ axes (see Fig. 3). Then some non-zero phase difference occurs between these components. The location of the maxima of the two-component interference patterns (e.g., the $x$ component) can remain unchanged, provided that the wedge and the polarization control of this interference pattern are adequately tuned. Consequently, the phase difference between the $y$ components of the superimposed beams is unavoidably shifted, resulting in a shift of the $y$-component interference pattern (Fig. 2b). The visibility of the resulting distribution becomes different from zero. Therefore, from the four polarization components involved in formation of the resulting field, only one polarization component undergoes a phase shift. The same effect (i.e., the change in the visibility of the resulting pattern) arises from changing ratio of amplitudes of the superimposing beams. This results in changing ratio of visibilities of the corresponding $x$ - and $y$-component interference patterns, without any relative phase shift between these components.

Ukr. J. Phys. Opt. 2007, V8, №4 

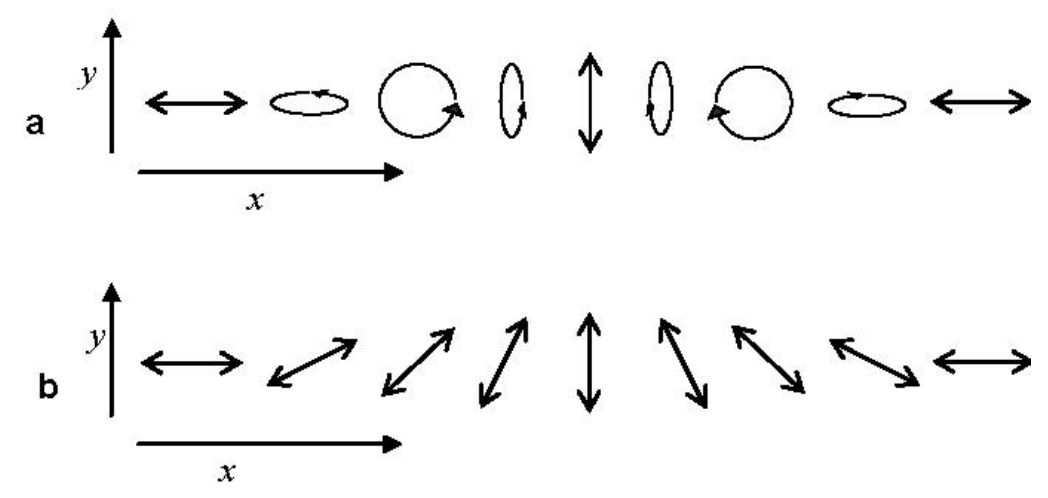

Fig. 4. Spatial period of polarization modulation of the resulting field for orthogonal linearly $\left( \pm 45^{\circ}\right)$ polarized waves from the slits (a) and orthogonal circularly polarized waves from the slits (b). In both cases, the intensity distribution at the observation plane is uniform, in consonance with Fig. $2 a$.

The two linearly polarized beams with the same polarization azimuths form a resulting field with a maximum visibility, where the maxima of interference patterns for the $x$ and $y$ components spatially coincide. In this case, the resulting visibility is affected only by the amplitude ratio for the two beams.

The gedanken experiment described above may be used for constructing elementary model for formation of the $S$ contours, which separate the nearest $C$ points with the opposite handedness for random, inhomogeneously polarized speckle fields [7]. So, if the waves of equal amplitudes at the slits 1 and 2 are circularly (clockwise and counterclockwise) polarized, then, again, one observes in the observation plane a uniform intensity distribution. Then the polarization modulation consists in gradually changing polarization azimuth of ubiquitously linearly polarized field (cf. Fig. 4b). The azimuth of polarization performs one complete revolution within the spatial period corresponding to the "scalar" interference pattern, which would occur if the polarization states at the two slits coincided with each other. This is merely a deterministic (model) analogue of the $S$ contour.

It seems predictable that the mean distance between the nearest points of a field, where the polarizations are mutually orthogonal (i.e., the average size of the $S$ contours, in terms of the topological approach), must also be comparable with the correlation length of this field. In framework of the statistical approach the latter is determined as a halfwidth of the function of mutual polarization. Changing the polarization state of the orthogonal component, while keeping the amplitude ratio of the two components constant, presumes the change by $\pi$ of the phase difference between the components. Naturally, the correlation length of polarization of the field should be close to the mean size of a speckle in a homogeneously polarized scalar speckle-field: $l_{\perp} \sim \lambda / \vartheta$, where $\lambda$ is the light wavelength and $\vartheta$ the angle of the field of view as seen from the observation plane. 


\section{Experiment}

Cuts of $100 \mu \mathrm{m}$-thick biological tissue (skin derma tissue), which strongly transform polarization of homogeneous probing beams, have been used as samples for our experimental studies. Surprisingly rich distribution of polarization states is formed in the boundary object field of such the samples due to birefringence, optical activity and phase delays of partial signals taking place in the bulk of the tissue [8]. It has been shown [9] that the mentioned samples have inhomogeneous structure. They consist of considerably birefringent islands occurring in optically isotropic bulk, which does not transform polarization state of the probing beam. Therefore, according to general principles, the state of polarization at any point of the boundary field is determined by the amplitude ratio and the phase differences of the partial beams. Then an optical system images the inhomogeneous boundary polarization field at the plane of analysis.

The optical arrangement for measuring coordinate distribution of the polarization parameters of samples is shown in Fig. 5 (see [9]). A collimated linearly polarized beam of $\mathrm{He}-\mathrm{Ne}$ laser (the diameter $10^{4} \mu \mathrm{m}$, the wavelength $\lambda=0.6328 \mu \mathrm{m}$ and the power $W=5.0 \mathrm{~mW}$ ) illuminates a sample. Polarization illuminator consisting of $\lambda / 4$-plates 3 and 5 and a polarizer 4 is used to form the probing beam with arbitrary polarization azimuths $\left(0^{\circ} \leq \alpha_{0} \leq 180^{\circ}\right)$ and ellipticities $\left(-45^{\circ} \leq \beta_{0} \leq+45^{\circ}\right)$.

The experimental conditions are provided so that to eliminate spatial-frequency filtration when imaging samples. This requires correspondence between the angular indicatrix characteristics of the light scattering by samples $\left(\Omega_{B T} \approx 16^{\circ}\right)$ and the angular aperture of objective of the microscope $\left(\Delta \omega=20^{\circ}\right)$. Here $\Omega_{B T}$ is the plane angle of cones wherein 98 per cent of the total scattered radiation energy is concentrated.

Inhomogeneously polarized images of samples are formed with a microobjective 7 at the plane of light-sensitive area of a CCD camera $10(800 \times 600$ pixels, each with the dimensions of $2 \mu \mathrm{m} \times 2 \mu \mathrm{m}$ ), which can probe structural elements of the samples in the range $2-2000 \mu \mathrm{m}$. Polarization analysis of the images is performed using a quarter-wave plate 8 and a linear polarizer 9 .

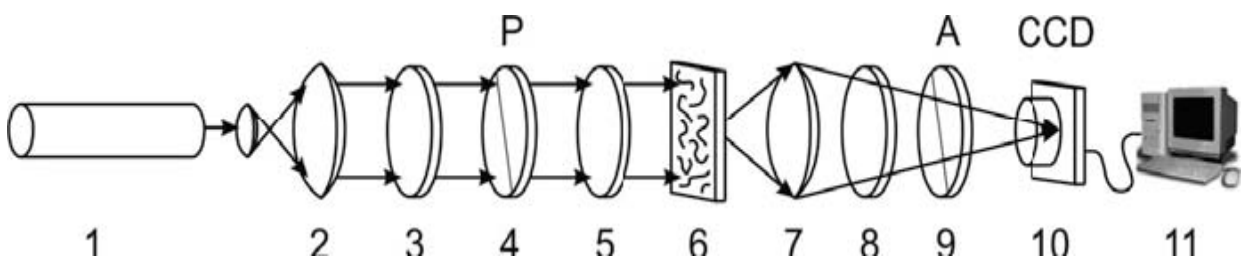

Fig. 5. Experimental setup: 1 - He-Ne laser; 2 -collimator; 3,5,8- quarter-wave plates; 4-polarizer; $6-$ a sample of biological tissue; 7-microobjective; 9 - analyzer; 10 - CCD camera; 11 - personal computer.

Ukr. J. Phys. Opt. 2007, V8, №4 
At first we measure the intensity distributions $I_{0}\left(r_{m, n}\right), I_{90}\left(r_{m, n}\right), I_{+45}\left(r_{m, n}\right)$ and $I_{-45}\left(r_{m, n}\right)$ for each pixel of CCD camera with the coordinates $r_{m, n}$ in the absence of quarter-wave plate, while orientating properly the transmittance axis of analyzer. Then we do the same for the intensity distributions $I_{r}\left(r_{m, n}\right)$ and $I_{l}\left(r_{m, n}\right)$, using the quarter-wave plate. As a result, one can form the tensors of the Stokes parameters,

$$
\begin{aligned}
& S_{0}\left(r_{m, n}\right)=I_{0}\left(r_{m, n}\right)+I_{90}\left(r_{m, n}\right), \quad S_{1}\left(r_{m, n}\right)=I_{0}\left(r_{m, n}\right)-I_{90}\left(r_{m, n}\right), \\
& S_{2}\left(r_{m, n}\right)=I_{+45}\left(r_{m, n}\right)-I_{-45}\left(r_{m, n}\right), \quad S_{3}\left(r_{m, n}\right)=I_{r}\left(r_{m, n}\right)-I_{l}\left(r_{m, n}\right),
\end{aligned}
$$

and find the tensors of normalized Stokes parameters, $s_{i}\left(r_{m, n}\right)=S_{i}\left(r_{m, n}\right) / S_{0}\left(r_{m, n}\right)$ $(i=0,1,2,3)$. Following a standard procedure, one determines tensors of the polarization azimuth, $\quad \alpha\left(r_{m, n}\right)=0.5 \arctan \left[s_{2}\left(r_{m, n}\right) / s_{1}\left(r_{m, n}\right)\right], \quad$ and the ellipticities, $\beta\left(r_{m, n}\right)=0.5 \arcsin s_{3}\left(r_{m, n}\right)$. In such a manner, one determines the areas (lines or points) with the linear and circular (clockwise or counterclockwise) polarizations, i.e. the polarization singularities. Our spatial resolution in the image plane $\left(500 \mathrm{~mm}^{-1}\right)$ is limited by a discrete structure of light-sensitive area of the CCD camera. This accuracy enables identifying localization of the polarization singularities. At the same time, the errors in determination of the Stokes parameters do not exceed 10 per cent.

Typical results of implementation of the mentioned procedure are presented in Fig. 6. They represent coordinate distribution of the Stokes parameter $S_{3}$ for one of the samples of skin derma. This parameter has been chosen for illustration as the most sensitive. Green colour in Fig. 6 corresponds to the areas with linear polarization $\left(S_{3}=0\right)$. There is an amorphous phase (optically isotropic bulk), which does not transform polarization state of the probing beam. The levels of red and blue correspond to the ellipticities characteristic respectively for the clockwise and counterclockwise polarizations, with the $C$ points of opposite signs located inside these areas. These are the areas with optically anisotropic (birefringent) inclusions.

In principle, one can process large fragments of the fields like that shown in Fig. 6 and then determine the mean size of the islands with clockwise of counterclockwise polarizations, which, in its turn, determines the correlation length of polarization parameters (or "polarization speckle"). A more efficient procedure performed by us consists in utilizing the measured intensities $I_{0}\left(r_{m, n}\right), I_{90}\left(r_{m, n}\right), I_{+45}\left(r_{m, n}\right), I_{-45}\left(r_{m, n}\right), I_{r}\left(r_{m, n}\right)$ and $I_{l}\left(r_{m, n}\right)$ for computing the coordinate distribution of the parameter $V$ in the image plane, following Eq. (1) for arbitrary sample intervals $\Delta r_{k}$. The advantages of this approach follow from a possibility for reconstructing spatial correlation function of the polarization parameters, of which half-width is the correlation length of the polarization structure. 


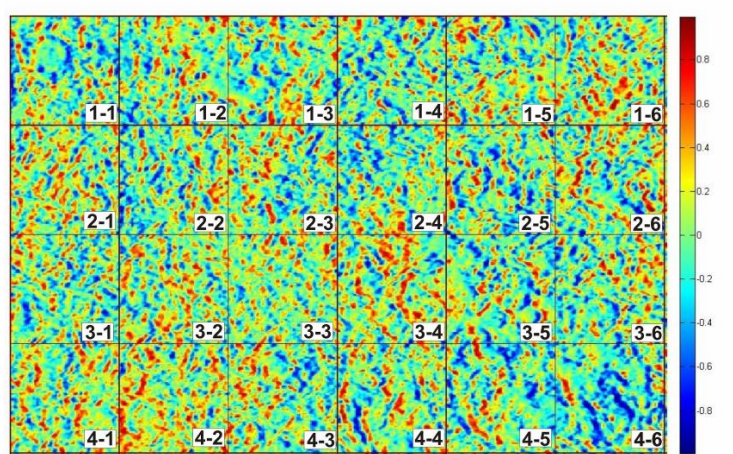

(a)

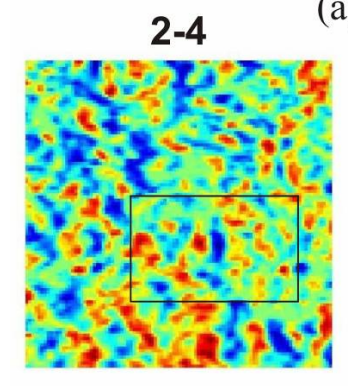

(b)

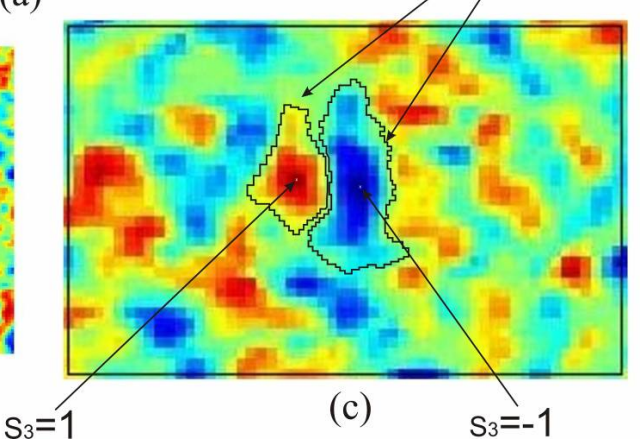

Fig. 6. Coordinate distribution of the Stokes parameters $S_{3}$ for the sample of skin derma (a); fragments (b) and (c) are the areas under analysis. The analyzed area (c) has the size $130 \mu \mathrm{m} \times 75 \mu \mathrm{m}$. The points where $s_{3}=+1$ and $s_{3}=-1$ are clockwise and counterclockwise $C$ points, respectively. Different colours correspond to different arguments of the sine function $s_{3}=\sin 2 \beta$.

We have computed the coordinate distribution of the parameter $V$ for the interval $\Delta r_{k}=1$ pixel. After that, we have computed two-dimensional autocorrelation function for the coordinate distribution of the parameter $V$ and determined its half-width. The results are shown in Fig. 7. The determined half-width for the parameter $V$ is $25 \mu \mathrm{m}$, with the experimental error $2 \mu \mathrm{m}$. This is in satisfactory agreement with the correlation length for this parameter $(\approx 20 \mu \mathrm{m})$ found within the topological approach as the average size of the $S$ contours.

The coordinate distribution of the polarization state in the boundary object field carries information on pre-history of this field and can be used for diagnostics of the object. It is especially important when one expects unambiguous interconnection of the parameters of the boundary field and the characteristics of the object illuminated by simple probing beam, as it takes place in our case of thin cuts of biological tissues. 


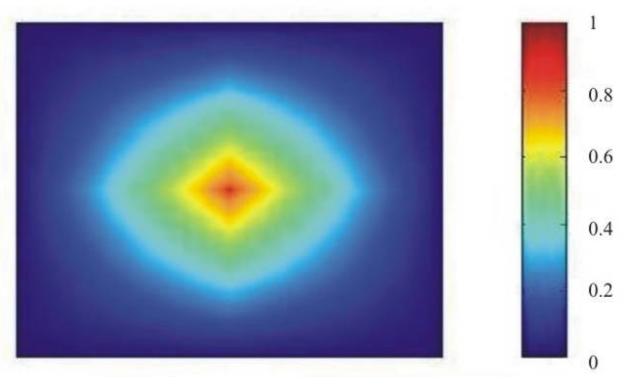

(a)

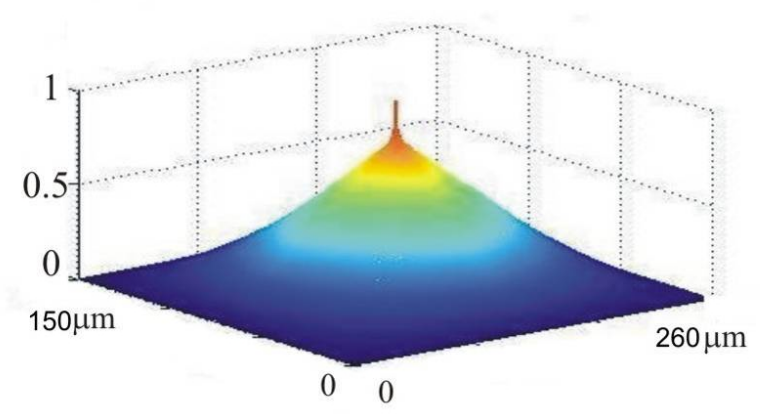

(b)

Fig. 7. Two-dimensional autocorrelation function for the coordinate distribution of the $V$ parameter for the area shown in Fig. 6c (a) and threedimensional presentation of this function (b). Different colours correspond to different magnitudes of the $V$ parameter.

Note that changes in the sample orientation or the polarization state of the probing beam result in considerable transformations of specific polarization structure of the field, so that the polarization singularities determining topology of the field change their location. Nevertheless, the correlation length of polarization parameters determined according to the algorithm of Eq. (1) remains invariable. That is why the statistical approach seems to be more preferable in the diagnostic problem, when compare to the topological approach.

\section{Conclusions}

We state that the correlation length of polarization of the field found from the degree of mutual polarization is comparable with the mean distance between the nearest points of the field with orthogonal states of polarization and it corresponds to the average size of the $S$ contours.

Coordinate distributions of the Stokes parameters characterize topology of polarization structure of the field, viz. enable determining locations of the $C$ points and the $S$ contours. Those distributions are helpful within the phenomenological approach describing inhomogeneously polarized fields. From the results of topological approach one can derive the averaged parameters (sizes) of the polarization distributions, such as the sizes of the $S$ contours or the mean distances between the $C$ points. 
This consideration leads to the conclusion that the statistical approach based on determination of the correlation function of mutual polarization seems to be more promising, in part in the problem of biomedical diagnostics. Besides, the correlation function of mutual polarization permits to conclude on the peculiarities of polarization changes within a polarization speckle (i.e., within a correlation length of polarization).

Generally, the statistical and topological approaches are complementary, providing the data on the spatial polarization structure both quantitatively (by estimating the degree of mutual polarization) and qualitatively (by determining the coordinate distributions of the Stokes parameters). To be unbiased, let us finally notice that the maps of coordinate distributions of the Stokes parameters can be also used for obtaining quantitative parameters that characterize correlation of the correlation parameters.

\section{References}

1. Angelsky O.V., Maksimyak P.P. and Hanson S.G. The Use of Optical-Correlation Techniques for Characterizing Scattering Object and Media. Bellingham: SPIE Press PM71 (1999).

2. Angelsky O.V. and Maksimyak P.P. Optical correlation diagnostics of surface roughness in: Optical Correlation Applications and Techniques. Bellingham: SPIE Press PM168 (2007).

3. Mokhun I.I. Introduction in linear singular optics in: Optical Correlation Applications and Techniques. Bellingham: SPIE Press PM168 (2007).

4. Wolf E, 2003. Unified theory of coherence and polarization of random electromagnetic beams. Phys. Lett A. 312: 263-267.

5. Tervo J, Setälä T and Friberg AT, 2003. Degree of coherence for electromagnetic fields. Opt. Exp. 11: 1137-1143.

6. Ellis J and Dogariu A, 2004. Complex degree of mutual polarization. Opt. Lett. 29: 536-538.

7. Angelsky OV, Mokhun AI, Mokhun II and Soskin MS, 2002. Interferometric methods in diagnostics of polarization singularities. Phys. Rev. E. 65: 036602(5).

8. Cowin SC, 2000. How is a tissue built? J. Biomed. Eng. 122: 553-568.

9. Angelsky OV, Ushenko AG, Ushenko YuA and Ushenko YeG, 2006. Polarization singularities of the object field of skin surface. J. Phys. D: Appl. Phys. 39: 35473558 .

Ukr. J. Phys. Opt. 2007, V8, №4 